\title{
Erratum to: Zero Preservation for a Family of Multivalued Functionals, and Applications to the Theory of Fixed Points and Coincidences
}

\author{
T. N. Fomenko ${ }^{a, *}$ and Yu. N. Zakharyan ${ }^{b, * *}$ \\ Presented by Academician of the RAS S.V. Matveev May 23, 2020 \\ Received May 23, 2020; revised May 23, 2020; accepted June 2, 2020
}

DOI: $10.1134 / \mathrm{S} 1064562421010087$

The sentence in p. 273 (left column, line 21 from below) should read as follows:

A multivalued functional $\Phi: Y \rightrightarrows \mathbb{R}_{+}$is called $(\alpha, \beta)$-search on $Y$ if, for any point $x \in Y$ and any $c \in \Phi(x)$ and $r>0$ such that $B(x, r) \subseteq Y$ and $c \leq(\alpha-\beta) r$, there exists a point $x^{\prime} \in Y$ and a value $c^{\prime} \in \Phi\left(x^{\prime}\right)$ for which $d\left(x, x^{\prime}\right) \leq \frac{1}{\alpha} c$ and $c^{\prime} \leq \frac{\beta}{\alpha} c$.

The second condition in Theorem 3 should read as follows:

(ii) For any $x \in \bar{U}$ and any $y \in T_{t}(x)$ and $r>0$ such that $B(x ; r) \subseteq \bar{U}$ and $d(y, Q) \leq(\alpha-\beta) r$, there exists a point $x^{\prime} \in \bar{U}$ and a value $y^{\prime} \in T_{t}\left(x^{\prime}\right)$ for which $\rho\left(x, x^{\prime}\right) \leq \frac{1}{\alpha} d(y, Q)$ and $d\left(y^{\prime}, Q\right) \leq \frac{\beta}{\alpha} d(y, Q)$.

All the results of the paper are correct. We sincerely apologize to the editorial board and the readers for the admitted inaccuracies.

The original article can be found online at https://doi.org/10.1134/S1064562420040225

\footnotetext{
${ }^{a}$ Faculty of Computational Mathematics and Cybernetics, Lomonosov Moscow State University, Moscow, 119991 Russia

${ }^{b}$ Faculty of Mechanics and Mathematics,

Lomonosov Moscow State University, Moscow, 119991 Russia

*e-mail:tn-fomenko@yandex.ru

**e-mail: yuri.zakharyan@gmail.com
} 